\title{
THE PREVALENCE OF ORO-FACIAL PAIN AND DISCOMFORT AMONG 16-YEAR OLD STUDENTS IN KELANTAN - A PILOT STUDY
}

Jaafar N, Saub R, Razak IA. The prevalence of oro-facial pain and discomfort among 16-year old students in Kelantan - a pilot study, Annals Dent Univ Malaya 1997; 4: 9-12

\begin{abstract}
A pilot study was conducted on 135 sixteen-year-old students from three rural schools in Kelantan to establish the prevalence of oro-facial pain and discomfort. About $44 \%$ reported to have experienced some oro-facial pain in the preceding four weeks. About $27 \%$ of those with pain, still experienced the pain at the time of clinical examination but only $8 \%$ have consulted professional help. Most of the pain encountered were only mild or moderate in nature. Only $7 \%$ and $10 \%$ respectively, reported that the pain affected their sleep and concentration to study. The main cause was toothache and sensitivity. The prevalence of discomfort was $22 \%$, the most common causes being recurrent oral ulcers and bleeding gums. The impacts of oro-facial pain was mainly manifested at the personal level, and very few affecting social functioning. However, untreated decay and missing teeth were very low (mean DT 0.47 , mean MT 0.27 ), while filled teeth (mean FT 2.9) was the main component of the DMFT (mean $3.66, \mathrm{sd} \pm 2.6$ ). Severe periodontal disease and the prevalence of traumatised teeth was not a major public health problem. The high prevalence of pain merit further research. Therefore a larger study involving other age-groups in other states is planned.
\end{abstract}

Key words: oral pain, oral discomfort, oral health survey.

\section{INTRODUCTION}

Most epidemiological studies conducted on schoolchildren in Malaysia have utilised 'objective' measures of disease levels such as the DMFT status, periodontal status and oro-facial anomalies (1-3). Although these data give valuable insights of disease trends to aid planning and evaluation of oral health programmes, they only provide us with a normative assessment of the situation. On the other hand, it is also important to evaluate the subjective point of view of oral conditions such as the impacts of oro-facial pain, discomfort and suffering as they affect the quality of life (4). For example, a person may have zero DMFT scores but may still suffer from oro-facial pain or discomfort from other sources. No studies have tried to link clinical indicators such as DMFT, CPITN and traumatised teeth to report oro-facial pain by the individual.

In Malaysia, very few studies have tried to collect subjective data on oro-facial pain. A survey involving adult factory workers reported $43.6 \%$ had suffered from oro-facial pain and/or discomfort over a four week period (5). In a military population in Kelantan, the overall prevalence over a four week period was about $52 \%$. This is despite the finding

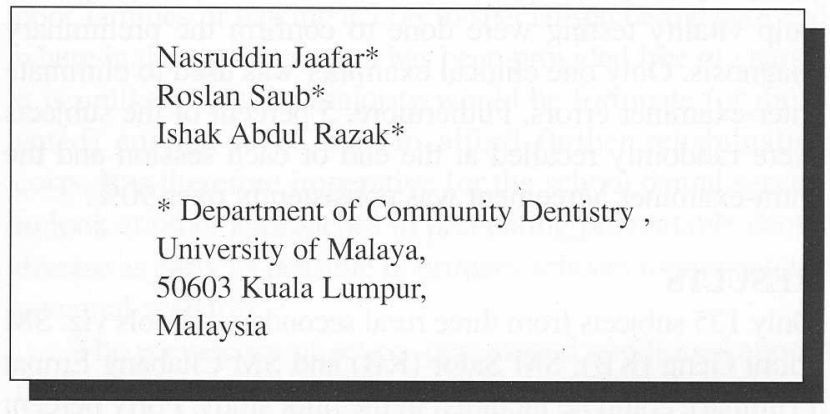

that the overall dental status of Army personnel, as measured by the DMFT index, was better than the civilian population (6). In a study involving the elderly population in Kelantan, dental sensitivity was found to be very prevalent affecting about $51 \%$ of the dentate subjects (7). Another study involving the elderly found that oral pain was the second most important reason for dental attendance after requests for denture (8).

Reisine (9) argued that oro-facial pain most frequently occurs in the younger age groups. However the literature on oro-facial pain among teenagers is very scarce. Realising the lack of subjective data in this population, a pilot study was carried out among 16-year-old students in selected schools in Kelantan.

The aim of the pilot study is to ascertain the prevalence of oro-facial pain and discomfort among teenage children. This data will be used as a baseline and the survey will serve to test the feasibility for carrying out a larger study. The objectives are (1) to establish the prevalence and type of orofacial pain and discomfort, (2) to describe the social impact, (3) to identify the clinical cause of the pain/discomfort, and (4) to estimate the level of dental disease using commonly used 'objective' measures (DMFT, CPITN, and traumatic injuries).

\section{MATERIALS AND METHOD}

The sampling frame consists of Form Four Malay students (average age 16 years) enrolled in government assisted schools in the Districts of Kota Bharu, Bachok, Pasir Putih, Pasir Mas, Tanah Merah and Tumpat in Kelantan. The first phase of selection was to select students who had continuous record of dental treatment by the School Dental Service since primary school. Unfortunately at this stage many schools had to be dropped out due to the problem of missing data. Of the schools selected to represent the various districts, only three schools met this criteria. All students with a continuous history of dental treatment were included in the sample.

The subjects were first asked to complete a self-administered questionnaire concerning episodes of pain and/or discomfort experienced in the past four weeks. This is fol- 
lowed by an interview to check on the accuracy of the responses about their oro-facial pain episodes. Subsequently the subjects were examined clinically to diagnose the cause of pain / discomfort and to record their dental caries status, periodontal status and presence of traumatised teeth. However no further investigations such as radiographs or pulp vitality testing were done to confirm the preliminary diagnosis. Only one clinical examiner was used to eliminate inter-examiner errors. Futhermore, 5 percent of the subjects were randomly recalled at the end of each session and the intra-examiner agreement was consistently over $90 \%$.

\section{RESULTS}

Only 135 subjects from three rural secondary schools viz. SM Pintu Geng (KB), SM Salor (KB) and SM Chabang Empat (Tumpat), could be included in the pilot study. Forty percent were males while the rest, females. About $75 \%$ of the subjects came from rural primary schools. Two-thirds had received treatment from mobile dental squads while the rest had static dental clinics located in their primary school. By the time they were in Primary 6 (12 years old), 73\% had completely received all their dental treatment needs (certified as 'completed cases').

Figure 1 shows the frequency according to the type of oro-facial pain reported. It should be noted that a subject may complain of one or more types of pain. Thus the total do not add-up to a hundred percent. The overall prevalence of oro-facial pain in the preceding four weeks was quite high $(44 \%)$. Toothache $(19 \%)$ and sensitivity to hot/cold foods or drinks (20\%), accounted for the most common pain complaints.

Of those with pain, $27 \%$ still felt the pain on the day of the clinical examination. Despite this very few $(8 \%)$ had made any attempt to consult a dentist or a dental health worker. The pain episodes in the last 4 weeks, (not including the survey day) ranged between 1-5 days (mean 1.8 days \pm 1.2 ). However the vast majority rated the pain as of mild or moderate severity with only occasional bursts of activity (98\%). Only two students were in continuously severe pain. About $7 \%$ reported they could not sleep well and $10 \%$ said it affected their concentration in class.

In the preceding 12 months, the majority $(67 \%)$ had experienced some form of oro-facial pain at some time or another. However only $16 \%$ were very concerned or worried about their oral health status.

Figure 2 shows the impact of the oro-facial pain. The most common impact was avoidance of certain foods or drinks which triggered the pain $(31 \%)$. A few tried to selfmedicate $(12 \%)$ with over-the-counter pain killers. These are impacts at the personal or individual level. The impact of oro-facial pain at the social level was, however, minimal. These include avoidance of friends and staying at home (less than 6\%).

Figure 3 shows the preliminary diagnoses of the cause of pain/discomfort. Ten percent of the 16-17 years old children in the study were found to have pain/discomfort caused by primary caries and further $7 \%$ by secondary caries. The second most important source of pain/discomfort were oral
Fig. 1 Prevalence \& type of oro-facial pain in 16yr-old Kelantan students in the previous 4 weeks

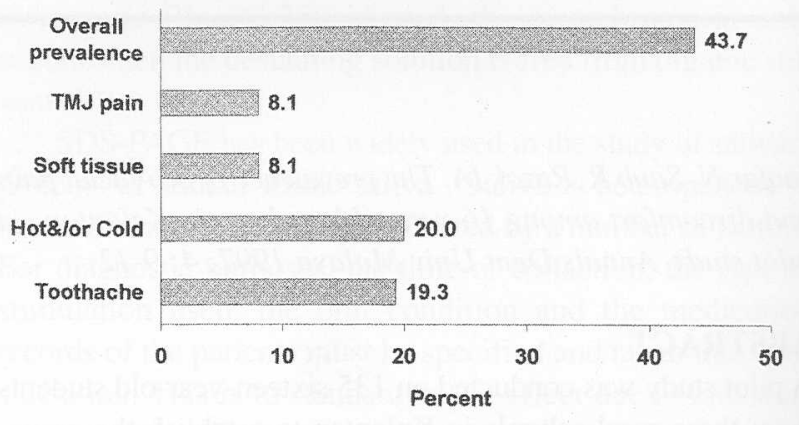

"Total do not add up to $100 \%$ because a subject may report one or more types of pain

Fig. 2 Social impacts of oro-facial pain in 16yr-old Kelantan students in the previous 4 weeks

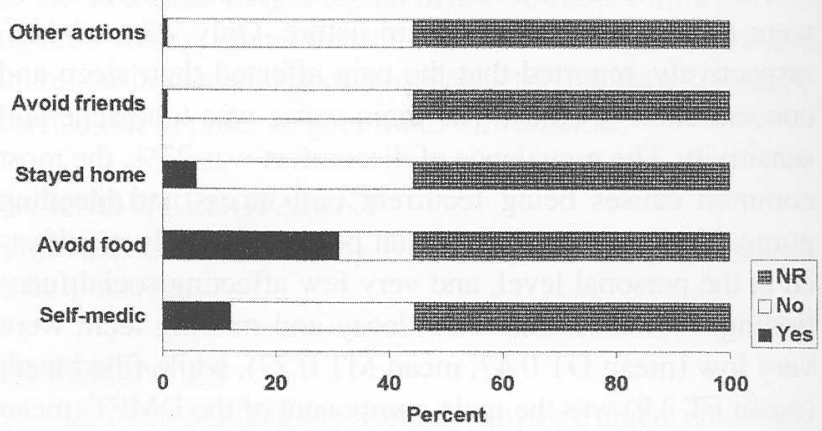

* NR- subject who did not report any pain

Fig. 3 Preliminary normative diagnosis of pain/discomfort based on clinical assessment on the day of examination

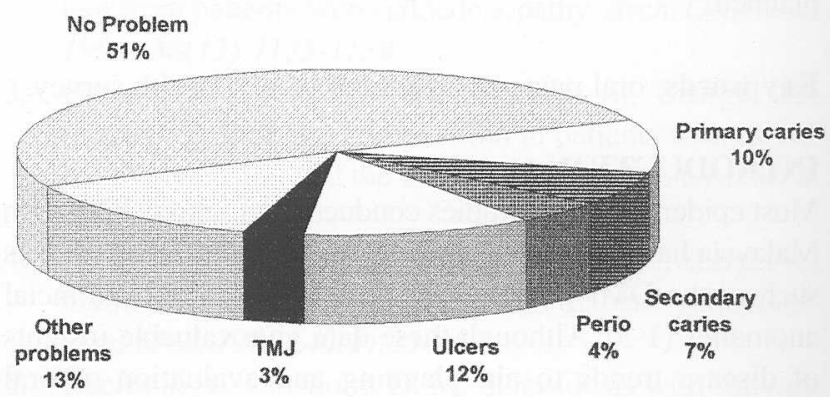

ulcers (12\%). Pain contributed by temporo-mandibular joint or periodontal problems were not significant.

The overall prevalence of discomfort in the previous 4 weeks was $22 \%(n=30)$. The most common causes of discomfort were recurrent oral ulcers $(11 \%)$ and bleeding gums $(11 \%)$. None of the subjects wore dentures. The discomfort were however of little impact and only $3 \%$ still had the discomfort on the day of examination.

The mean DMFT of the sample was $3.66(\mathrm{SD} \pm 2.6)$. The main component of the DMF was contributed by FT (mean $2.9 \pm 2.2$ ) while DT (mean 0.47) and MT (mean 0.27) were obviously very low. An estimate of the cost of treatment was based on the lowest price, in the range of prices as recommended by the Malaysian Private Practitioners Association which reflects the market value of restorative 
treatment, shows that each student had received on average restorative treatment worth about RM 90 (SD \pm RM 65). However the need for further restorative treatment, assuming ideal conditions are met (for eg. including endodontic needs, crowns and bridges), was estimated to cost on average about RM 340 per student ( \pm RM 755).

Periodontal disease did not seem to be a major public health problem since almost $70 \%$ of subjects had all their quadrants scoring CPITN $=0$. Only about $10 \%$ had one or more gingival bleeding unit and $25 \%$ had some calculus present.

The prevalence of traumatised teeth were also quite low $(5.2 \%, n=7)$. All subjects affected had only one tooth traumatized and only one student had a severe fracture which affected two-thirds of the crown. However, no vitality tests were done to confirm the severity.

\section{DISCUSSION}

The total number of subjects who qualified for the inclusion criteria was relatively small $(n=135)$. This has two implications. First the results cannot be generalised. Second, further studies which need to rely on past dental records among 16year olds in Kelantan had to be ruled out due to lack of records. It should therefore be emphasized that dental records of schoolchildren should be kept in continuity from primary through secondary schools in order to keep track of individual progress and for future evaluation. Unfortunately this pilot study in Kelantan found that many records were lost through vermin, improper storage and handling especially during the crucial period when students were shifting from primary to secondary schools. Thus a valuable source of retrospective data were lost.

Nevertheless from the data available, the mean DMFT of 3.66 at 16 years of age indicates a commendable achievement, especially considering the low level of untreated decay and missing teeth. Filled teeth contributed the most to the DMFT index, indicating a very successful restorative service run by the school dental service. This level is comparatively lower than the National survey done in 1988 (mean DMFX 4.86)(1). However as cautioned earlier, the small sample size and the exclusion of so many potential subjects makes this a speculative assumption. A larger study is needed to confirm this.

Despite the long history of dental treatment and the fact that $73 \%$ of the students had been certified as dentally fit when they left primary school at the age of 12 years old, the prevalence of oro-facial pain and discomfort is quite high, affecting almost one in two subjects (44\%). This level is comparable to those found among adult factory workers (5) and just slightly lower than the prevalence in a military population (6). It is noteworthy that despite the favourable rate of met restorative treatment needs, oro-facial pain and suffering is still common. There is therefore a need to address or treat other sources of pain besides merely treating dental caries in the School Dental Service.

The second implication of the high rate of restorations is the cost. While the present mean cost of treatment received (using the Private Practitioners Recommended Fee Schedule as a guide), appears to be acceptable at around RM 90 per child throughout their school years, the estimated future treatment needs, at market rates, to achieve restorative ideals (including endodontic needs, crowns and bridges), were quite high ranging from RM 25 to over RM 3500 (mean RM 340). Being a rural population of which the majority are from poor families or lacking access to specialised dental care and where in the past, treatment has been provided free of charge, it is unlikely that the majority would be fortunate (or motivated) enough to be able to afford further rehabilitation costs. It is therefore imperative for the school dental service to look at other approaches in preventing preventable dental disease as early as possible in primary schools to prevent this potential scenario.

The prevalence of severe periodontal conditions appear to be well under control. Most of the treatment needs do not go beyond the need for effective oral hygiene and scaling. This may be attributed to the vigorous dental health education campaigns frequently aimed at schoolchildren in Kelantan and improved standards of living. Similarly the prevalence of traumatised teeth (around 5\% ) is not thought as a major public health problem. However as stated earlier, a more comprehensive oral health promotion strategy to inculcate healthier lifestyle is needed besides the limited dental health education programmes.

In conclusion, it may be said that oro-facial pain and discomfort is an important public health issue as it affects almost one in two persons in the population. This is despite the fact that the majority of restorative dental needs have been met. Although not extremely severe in most cases, the impact at the individual level is significant and by extension, the impact on the quality of life (10). Since most of these conditions are preventable or treatable, further studies are planned as follow-up elsewhere and involving a different age-group. Missing dental records has been identified in this pilot study as one of the major problems to be overcome. The data will be very useful in adding further dimension in evaluating the impact of our oral health programmes in the target population.

\section{ACKNOWLEDGEMENTS}

The study was supported by a Research Grant from University Malaya (No. PJP 107/94) - with the co-operation of the Research Division (Schools) Ministry of Education and Dental Division Ministry of Health. We wish to express our gratitude to support staff En. Zamri of Kota Bharu Dental Clinic, Dr Roslan A. Rahman; Dr Abdul Rani, Dr Nizam Abdullah and all dental officers and staff of the schools involved and the Kelantan Education Department.

\section{REFERENCES}

1. Dental Division. Dental epidemiological survey of school children in Peninsular Malaysia 1988. Kuala Lumpur: Dental Division Ministry of Health Malaysia 1988.

2. Dental Division. Dental epidemiological survey of school children in Sarawak Nov. 1980 - Nov. 1981. Kuala Lumpur: Dental Division Ministry of Health Malaysia 1982. 
3. Dental Division. Dental epidemiological survey of school children in Sabah July 1985 - October 1985. Kuala Lumpur: Dental Division Ministry of Health Malaysia 1986.

4. Locker D, Miller Y. Evaluation of subjective oral health indicators. J Public Health Dent 1994; 54: 167-76.

5. Jaafar N, Razak IA, Zain RB. The social impact of oral and facial pain in an industrial population. Annals Academy Medicine Singapore 1989; 18: 553-5.

6. Nik Mohd Zukri WH. Prevalence and impact of orofacial pain and discomfort among Malaysian army personnel. Kuala Lumpur: Dept of Community Dent Univ Malaya, MCD thesis 1996.
7. Noralaini I. Tooth loss and perception of oral function of an elderly Malay population in Kelantan. Kuala Lumpur: Dept of Community Dent Univ Malaya, MCD thesis 1996.

8. Razak IA, Ali MM. Need and demand for dental care in a group of Malaysian elderly. Gerodontics 1988; 4: 265-7.

9. Reisine ST. Dental health and public policy: the social impact of dental disease. Am J Public Health 1985; 75: 27-30.

10. Reisine ST. The effect of pain and oral health on the quality of life. Community Dent Health 1988; 5: 63-8. 